MATHEMATICS OF COMPUTATION

Volume 73 , Number 245 , Pages 451-473

S 0025-5718(03)01556-4

Article electronically published on June 11, 2003

\title{
THE HEXAGONAL VERSUS THE SQUARE LATTICE
}

\author{
PIETER MOREE AND HERMAN J.J. TE RIELE
}

\begin{abstract}
Schmutz Schaller's conjecture regarding the lengths of the hexagonal versus the lengths of the square lattice is shown to be true. The proof makes use of results from (computational) prime number theory.

Using an identity due to Selberg, it is shown that, in principle, the conjecture can be resolved without using computational prime number theory. By our approach, however, this would require a huge amount of computation.
\end{abstract}

\section{INTRODUCTION}

In [17, p. 201] Schmutz Schaller, motivated by considerations from hyperbolic geometry, makes the conjecture that in dimensions 2 to 8 the best known lattice sphere packings have 'maximal lengths', that is, that their length spectrum dominates the length spectrum of every other lattice of the same dimension and covolume at every position, and goes on to write: "In dimension 2 the conjecture means in particular that the hexagonal lattice is 'better' than the square lattice. More precisely, let $0<h_{1}<h_{2}<\cdots$ be the positive integers, listed in ascending order, which can be written as $h_{i}=x^{2}+3 y^{2}$ for integers $x$ and $y$. Let $0<q_{1}<q_{2}<\cdots$ be the positive integers, listed in ascending order, which can be written as $q_{i}=x^{2}+y^{2}$ for integers $x$ and $y$. Then the conjecture is that $q_{i} \leq h_{i}$ for $i=1,2,3, \ldots$." That he uses the words 'in particular' is a bit surprising since the conjecture for dimension 2 implies that if a plane lattice having the same covolume as the hexagonal lattice $\Sigma$ fails to be isometric to $\Sigma$, then its length spectrum is dominated by that of $\Sigma$ at every position, which is weaker than the conjecture between the quotation marks, which asserts that the length spectrum of $\Sigma$, thought of as the Eisenstein numbers, dominates that of the square lattice in its realization as Gaussian integers (note that the Eisenstein numbers have smaller covolume than the Gaussian integers).

The reader might also be surprised to see the norm form $x^{2}+3 y^{2}$ appearing in the quotation, rather than $x^{2}+x y+y^{2}$. However, both represent the same integers. Notice that $x^{2}+3 y^{2}$ is the norm form of the sublattice $\left[1,2 \zeta_{3}\right]$ of index 2 of $\Sigma$. The two other sublattices of index 2 are easily seen to be $\left[2, \zeta_{3}\right]$ and $\left[-1+\zeta_{3}, 1+\zeta_{3}\right]$. As under multiplication by $\zeta_{3}$ the sublattices are transformed into each other, they each have the same length spectrum. Since the union of the three sublattices is $\Sigma$, the length spectrum of every sublattice of index 2 must be the same as that of $\Sigma$ itself.

For a more introductory account to Schmutz Schaller's work than [17, see [18]. For some progress regarding Schmutz Schaller's general conjecture in dimension 2 see [8] (this case of the conjecture is also mentioned in 4]).

Received by the editor May 2, 2002 and, in revised form, August 6, 2002.

2000 Mathematics Subject Classification. Primary 11N13, 11Y35, 11Y60. 
For $j \geq 1$ let $b_{j}(n)=1$ if $n$ is represented by the quadratic form $X^{2}+j Y^{2}$ and $b_{j}(n)=0$ otherwise. The characteristic functions $b_{1}$ and $b_{3}$ are well understood. The following result was already known to Fermat.

Lemma 1. A positive integer $n$ is represented by the form $X^{2}+Y^{2}$ if and only if every prime factor $p$ of $n$ of the form $p \equiv 3(\bmod 4)$ occurs to an even power. A positive integer $n$ is represented by the form $X^{2}+3 Y^{2}$ if and only if every prime factor $p$ of $n$ of the form $p \equiv 2(\bmod 3)$ occurs to an even power.

(In general the natural numbers $n$ that are represented by a quadratic form $X^{2}+m Y^{2}$ are rather more difficult to describe, cf. the beautiful book of D. Cox 5].) Lemma 1 implies that $b_{1}$ and $b_{3}$ are multiplicative functions.

Let $B_{i}(x)=\sum_{n \leq x} b_{i}(n)$ for $i=1$ and $i=3$. Schmutz Schaller's conjecture regarding the square versus the hexagonal lattice can be reformulated as follows in terms of $B_{1}$ and $B_{3}$.

Conjecture 1. We have $B_{1}(x) \geq B_{3}(x)$ for every $x$.

The first asymptotic result on $B_{1}(x)$ goes back to Landau [9], who proved in 1908 that

$$
B_{1}(x) \sim C_{b_{1}} \frac{x}{\sqrt{\log x}},
$$

where

$$
C_{b_{1}}=\frac{1}{\sqrt{2}} \prod_{p \equiv 3(\bmod 4)}\left(1-p^{-2}\right)^{-1 / 2}=\frac{\pi}{4} \prod_{p \equiv 1(\bmod 4)}\left(1-p^{-2}\right)^{1 / 2} \approx 0.764 .
$$

(Here and in the sequel the letter $p$ is used to indicate primes.) Landau's proof uses contour integration. It is not difficult to use his method to show, cf. [20], that for every $k \geq 2$ there exist constants $C_{b_{1}}(2), \ldots, C_{b_{1}}(k)$ such that

$$
B_{1}(x)=C_{b_{1}} \frac{x}{\sqrt{\log x}}\left(1+\frac{C_{b_{1}}(2)}{\log x}+\cdots+\frac{C_{b_{1}}(k)}{\log ^{k-1} x}+O\left(\frac{1}{\log ^{k} x}\right)\right) .
$$

This result can also be established by methods not using complex analysis, cf. [14] p. 288]. At the beginning of 1913 a then unknown Hindu clerk by the name of Ramanujan wrote in his first letter to Hardy [2] that he could prove that

$$
B_{1}(x)=C_{b_{1}} \int_{2}^{x} \frac{d t}{\sqrt{\log t}}+O\left(x^{1-\varepsilon}\right),
$$

for some $\varepsilon>0$. (For a reconstruction of Ramanujan's speculative argument see 1, pp. 60-66].) Note the similarity of Ramanujan's claim with the prime number theorem. From (4) we infer that $C_{b_{1}}(2)=1 / 2$ by partial integration. Shanks [21] showed, however, that $C_{b_{1}}(2) \neq 1 / 2$, thus disproving Ramanujan's claim. (Ramanujan gave the correct formula and numerical approximation for $C_{b_{1}}$, though.) The constants $C_{b_{1}}$ and $C_{b_{1}}(2)$ are known as the Landau-Ramanujan constant and the second order Landau-Ramanujan constant, respectively. For more on the evaluation of these constants see Section [5] For more on mathematical constants in general, see, e.g., [7].

Ramanujan [3] stated several claims similar to (4) in his 'unpublished' manuscript on the partition and tau functions, see Section 6. All of them are disproved in [11]. It can be shown, however, that in each case Ramanujan's claims give the correct asymptotic main term. 
TABLE $1 . B_{1}(x)$ versus $B_{3}(x)$

\begin{tabular}{|c|c|c||c|c|c|}
\hline$x$ & $B_{1}(x)$ & $B_{3}(x)$ & $x$ & $B_{1}(x)$ & $B_{3}(x)$ \\
\hline \hline $2^{1}$ & 2 & 1 & $2^{14}$ & 4357 & 3645 \\
\hline $2^{2}$ & 3 & 3 & $2^{15}$ & 8363 & 6993 \\
\hline $2^{3}$ & 5 & 4 & $2^{16}$ & 16096 & 13456 \\
\hline $2^{4}$ & 9 & 8 & $2^{17}$ & 31064 & 25978 \\
\hline $2^{5}$ & 16 & 14 & $2^{18}$ & 60108 & 50248 \\
\hline $2^{6}$ & 29 & 25 & $2^{19}$ & 116555 & 97446 \\
\hline $2^{7}$ & 54 & 45 & $2^{20}$ & 226419 & 189291 \\
\hline $2^{8}$ & 97 & 82 & $2^{21}$ & 440616 & 368338 \\
\hline $2^{9}$ & 180 & 151 & $2^{22}$ & 858696 & 717804 \\
\hline $2^{10}$ & 337 & 282 & $2^{23}$ & 1675603 & 1400699 \\
\hline $2^{11}$ & 633 & 531 & $2^{24}$ & 3273643 & 2736534 \\
\hline $2^{12}$ & 1197 & 1003 & $2^{25}$ & 6402706 & 5352182 \\
\hline $2^{13}$ & 2280 & 1907 & $2^{26}$ & 12534812 & 10478044 \\
\hline
\end{tabular}

Similarly to (3), it can be shown that for arbitrary $k \geq 2$ there exist constants $C_{b_{3}}(2), \ldots, C_{b_{3}}(k)$ such that

$$
B_{3}(x)=C_{b_{3}} \frac{x}{\sqrt{\log x}}\left(1+\frac{C_{b_{3}}(2)}{\log x}+\cdots+\frac{C_{b_{3}}(k)}{\log ^{k-1} x}+O\left(\frac{1}{\log ^{k} x}\right)\right),
$$

where

$C_{b_{3}}=\frac{1}{\sqrt{2}} \frac{1}{3^{1 / 4}} \prod_{p \equiv 2(\bmod 3)}\left(1-p^{-2}\right)^{-1 / 2}=\frac{\pi 3^{1 / 4} \sqrt{2}}{9} \prod_{p \equiv 1(\bmod 3)}\left(1-p^{-2}\right)^{1 / 2} \approx 0.639$.

We thus arrive at the following conclusion.

Proposition 1. Conjecture 1 is asymptotically true.

Table 1 (copied from 22] and verified by the second author) suggests that Conjecture 1 is true for small $x$ as well. The literature thus provides us with good indications that Conjecture 1 is true. The purpose of this paper is to go beyond this and prove that Conjecture 1 is indeed true.

Theorem 1. We have $B_{1}(x) \geq B_{3}(x)$ for every $x$. That is, Schmutz Schaller's conjecture that the hexagonal lattice is 'better' than the square lattice is true.

Landau's classical result (1) has been generalised in many directions; see [12] for a survey with over 50 references. Despite this rich history, nobody but the first of the present authors (in [10]) seems to have been concerned with proving effective results in this area, which is precisely what is needed to establish Theorem 1

\section{Preliminaries}

Let $f$ be a multiplicative function from the natural numbers to $\mathbb{R}_{>0}$. We define $M_{f}(x)=\sum_{n \leq x} f(n), \mu_{f}(x)=\sum_{n \leq x} f(n) / n$ and $\lambda_{f}(x)=\sum_{n \leq x} f(n) \log n$. We denote the formal Dirichlet series $\sum_{n=1}^{\infty} f(n) n^{-s}$ associated to $f$ by $L_{f}(s)$. We define $\Lambda_{f}(n)$ by

$$
-\frac{L_{f}^{\prime}(s)}{L_{f}(s)}=\sum_{n=1}^{\infty} \frac{\Lambda_{f}(n)}{n^{s}}
$$


Notice that

$$
f(n) \log n=\sum_{d \mid n} f(d) \Lambda_{f}\left(\frac{n}{d}\right) .
$$

If $f$ is the characteristic function of a multiplicative subsemigroup of the natural integers with $(1<) q_{1}<q_{2}<\cdots$ as generators, then it can be shown that $\Lambda_{f}(n)=$ $\log q_{i}$ if $n$ equals a positive power of a generator $q_{i}$, and $\Lambda_{f}(n)=0$ otherwise. For $f=b_{1}$ we thus find, using Lemma 1,

$$
\Lambda_{b_{1}}(n)= \begin{cases}2 \log p & \text { if } n=p^{2 r}, r \geq 1 \text { and } p \equiv 3(\bmod 4) \\ \log p & \text { if } n=p^{r}, r \geq 1 \text { and } p \equiv 1(\bmod 4) \text { or } p=2 \\ 0 & \text { otherwise. }\end{cases}
$$

For $f=b_{3}$ we find

$$
\Lambda_{b_{3}}(n)= \begin{cases}2 \log p & \text { if } n=p^{2 r}, r \geq 1 \text { and } p \equiv 2(\bmod 3) \\ \log p & \text { if } n=p^{r}, r \geq 1 \text { and } p \equiv 1(\bmod 3) \text { or } p=3 \\ 0 & \text { otherwise. }\end{cases}
$$

From property (6) of $\Lambda_{f}(n)$, we easily infer that

$$
\lambda_{f}(x)=\sum_{n \leq x} f(n) \psi_{f}\left(\frac{x}{n}\right),
$$

where $\psi_{f}(x)=\sum_{n \leq x} \Lambda_{f}(n)$. The functions $\Lambda_{f}$ and $\psi_{f}$ are analogues of, respectively, the von Mangoldt function and the Chebyshev $\psi$-function.

\section{Some Related CONJECTURES}

Unfortunately it seems that $M_{f}$ is not a very natural mathematical object, whereas $\mu_{f}$ is (as is amply demonstrated by browsing through the literature). For this reason we consider two additional conjectures:

Conjecture 2. We have $\lambda_{b_{1}}(x) \geq \lambda_{b_{3}}(x)$ for $x \geq 8$.

Conjecture 3. We have $\mu_{b_{1}}(x) \geq \mu_{b_{3}}(x)$ for every $x$.

Note that $\exp \left(\lambda_{b_{1}}(x) / 2\right)$ is the product of all different lengths in the square lattice not exceeding $\sqrt{x}$. Thus Conjecture 2 can be reformulated as stating that the product of the different distances not exceeding $x$ occurring in the square lattice always exceeds the product of the different distances not exceeding $x$ in the hexagonal lattice, provided that $x \geq 2 \sqrt{2}$.

Conjecture 1 clearly implies Conjecture 3. Furthermore we have:

Proposition 2. Conjecture 2 implies Conjecture 1.

Proof. We have, for $x \geq 2$,

$$
M_{f}(x)=\int_{2-}^{x} \frac{d \lambda_{f}(t)}{\log t}=\frac{\lambda_{f}(x)}{\log x}+\int_{2}^{x} \frac{\lambda_{f}(t)}{t \log ^{2} t} d t .
$$

Denote the latter integral by $I_{f}(x)$. It is not difficult to show that $I_{b_{1}}(x) \geq I_{b_{3}}(x)$ for $x \leq 8$. Conjecture 2 then implies that the latter inequality holds for every $x$. The truth of Conjecture 2 together with (8) then implies that $B_{1}(x) \geq B_{3}(x)$ for $x \geq 8$. By direct computation we then infer that the latter inequality holds for every $x$. 
Thus in order to establish Theorem 1, it suffices to establish Conjecture 2. From (71) and $\psi_{b_{i}}(x) \sim x / 2$ as $x$ tends to infinity it follows that $\lambda_{b_{i}}(x) \sim \mu_{b_{i}}(x) / 2$, as $x$ tends to infinity. An effective form of this relationship, together with an effective estimate for $\mu_{b_{i}}$ (provided by Lemma 2), then allows us to prove the main result of this paper:

Theorem 2. Conjectures 1,2 and 3 are all true.

Complications arise due to the fact that

$$
\lim _{x \rightarrow \infty} B_{1}(x) / B_{3}(x)=1.1961377420 \cdots,
$$

which is rather close to 1 , and that $\psi_{b_{i}}(y)$ is not so close to $y / 2$ for various ranges of small $y$ (the convolutional nature of (7) forces us to take the small $y$ range into account).

\section{The TOOLBox}

The following result from [10] will play a crucial rôle. It is in essence an effective version of Theorem A of [25].

Lemma 2. Let $f$ be a multiplicative function from the natural numbers to $\mathbb{R}_{\geq 0}$. Suppose that there exist constants $D_{-}, D_{+}$and $\tau$, with $\tau>0$, such that for every $x \geq x_{0}$,

$$
D_{-} \mu_{f}(x) \leq \sum_{n \leq x} \frac{f(n)}{n}\left\{\sum_{m \leq \frac{x}{n}} \frac{\Lambda_{f}(m)}{m}-\tau \log \frac{x}{n}\right\} \leq D_{+} \mu_{f}(x) .
$$

Then we have, for $x>\max \left\{x_{0}, \exp \left(D_{+}\right)\right\}$,

$$
\frac{C_{f}}{\tau} \log ^{\tau} x \frac{\left(1-\frac{D_{+}}{\log x}\right)^{\tau+1}}{1-\frac{D_{-}}{\log x}} \leq \mu_{f}(x) \leq \frac{C_{f}}{\tau} \log ^{\tau} x \frac{\left(1-\frac{D_{-}}{\log x}\right)^{\tau+1}}{1-\frac{D_{+}}{\log x}},
$$

where

$$
C_{f}:=\frac{1}{\Gamma(\tau)} \lim _{s \rightarrow 1+0}(s-1)^{\tau} L_{f}(s) .
$$

In particular, if there exist constants $C_{-}$and $C_{+}$such that

$$
C_{-} \leq \sum_{n \leq x} \frac{\Lambda_{f}(n)}{n}-\tau \log x \leq C_{+} \text {for } x \geq 1,
$$

then (10) holds true, for $x>\exp \left(C_{+}\right)$, with $D_{-}=C_{-}$and $D_{+}=C_{+}$.

Remark 1. From the proof of this lemma, $\int_{1}^{x} \mu_{f}(t) d t / t$ appears as a more easily estimated function than $\mu_{f}(x)$. Interestingly, Landau [9] in his proof of (1) using contour integration, estimates $\int_{1}^{x} \mu_{b_{1}}(t) d t / t$ rather than $B_{1}(x)$ itself.

Remark 2. If $\lim _{x \rightarrow \infty}\left(\sum_{n \leq x}\left(\Lambda_{f}(n) / n-\tau \log x\right)\right)$ exists, we denote it by $B_{f}$.

Let us put

$$
L\left(x, \tau, D_{-}, D_{+}\right)=\frac{\left(\log x-D_{+}\right)^{\tau+1}}{\log x-D_{-}} \text {and } U\left(x, \tau, D_{-}, D_{+}\right)=\frac{\left(\log x-D_{-}\right)^{\tau+1}}{\log x-D_{+}} .
$$

Thus we can write (10) as $C_{f} L\left(x, \tau, D_{-}, D_{+}\right) / \tau \leq \mu_{f}(x) \leq C_{f} U\left(x, \tau, D_{-}, D_{+}\right) / \tau$. 
Let $r, s$ and $c_{1}$ be given. At a few instances in the sequel we want to show that for every $x \geq x_{2}$, with $x_{2}$ some explicit constant, we have $\mu_{f}(x / r) \geq c_{1} \mu_{g}(x / s)$, where $g$ satisfies the conditions of Lemma 2 with constants $\tau, D_{-}^{\prime}$ and $D_{+}^{\prime}$. By Lemma 2 this leads us to consider inequalities of the form

$$
L\left(\frac{x}{r}, \tau, D_{-}, D_{+}\right) \geq c_{2} U\left(\frac{x}{s}, \tau, D_{-}^{\prime}, D_{+}^{\prime}\right),
$$

where all variables and constants are real numbers with $\tau, r, s$ and $c_{2}$ positive, $D_{-} \leq D_{+}, D_{-}^{\prime} \leq D_{+}^{\prime}$ and $x \geq x_{0}:=\max \left\{\exp \left(D_{+}^{\prime}\right) s, \exp \left(D_{+}\right) r\right\}$. We recall the following lemma from [10]:

Lemma 3. If $\log s+D_{-}^{\prime} \leq D_{+}+\log r$ and (13) is satisfied for some $x_{1}>x_{0}$, then (13) is satisfied for every $x \geq x_{1}$. If $\log s+D_{-}^{\prime}>D_{+}+\log r$ and

$$
c_{2}\left(1+\frac{D_{+}^{\prime}-D_{-}^{\prime}}{\log \left(x_{1} / s\right)-D_{+}^{\prime}}\right) \leq 1+\frac{D_{-}-D_{+}}{\log \left(x_{1} / r\right)-D_{-}}
$$

for some $x_{1}>x_{0}$, then (13) is satisfied for every $x \geq x_{1}$.

We also need the following result about the difference between $U\left(\frac{x}{r}, \frac{1}{2}, D_{-}, D_{+}\right)$ and $L\left(\frac{x}{s}, \frac{1}{2}, D_{-}, D_{+}\right)$.

Lemma 4. Assume that $D_{+}>D_{-}$and $s \geq r \geq 1$. The difference

$$
U\left(\frac{x}{r}, \frac{1}{2}, D_{-}, D_{+}\right)-L\left(\frac{x}{s}, \frac{1}{2}, D_{-}, D_{+}\right)
$$

is monotonically decreasing for $x \geq s \exp \left(1.01 D_{+}-0.01 D_{-}\right)$.

The difference in the latter lemma multiplied by $C_{b_{i}}$ appears if we try to bound $\mu_{b_{i}}(x / r)-\mu_{b_{i}}(x / s)$ from above. Notice that the latter difference is not monotonically decreasing from any $x$ onwards, although it can be bounded above by a function that is monotonically decreasing for all sufficiently large $x$.

Our proof of Lemma 4 uses the following lemma.

Lemma 5. Let $y$ and $\delta$ be non-negative real numbers. Then the inequality

$$
\sqrt{y+1+\delta}(y+\delta-2)(y+1)^{2} \leq \sqrt{y}(y+3)(y+\delta)^{2}
$$

holds if either $\delta \leq 2$ or $y \geq 0.0099945$.

Proof. On replacing the inequality sign in (14) with the equality sign and squaring both sides, we obtain an equation of an algebraic curve. Using continuity and, e.g., Maple's function fsolve (for numerically determining roots of polynomial equations), the result can then be deduced.

Remark. For $y=0.0099944$ and $\delta \approx 5.4$ inequality (14) is not satisfied. Indeed, if we square both sides of the inequality and take the difference, then, considered as a polynomial in $y$, the discriminant has $27 \delta^{5}-198 \delta^{4}+410 \delta^{3}-936 \delta^{2}+1299 \delta-730$ as a factor, which has $5.44694735 \cdots$ as its largest real root. Considered as a polynomial in $\delta$, we find

$$
27 y^{8}-72 y^{7}-2380 y^{6}-12792 y^{5}-33822 y^{4}-48888 y^{3}-32076 y^{2}-2376 y+27
$$

as a factor of the discriminant, which has $0.00999445028 \cdots$ as its next to largest real root.

We can now prove Lemma 4. 
Proof of Lemma 4. Differentiating $U\left(x / r, \frac{1}{2}, D_{-}, D_{+}\right)-L\left(x / s, \frac{1}{2}, D_{-}, D_{+}\right)$yields, after some tedious calculations, that the derivative is non-positive provided that (14) is satisfied with

$$
y=\left(\log (x / s)-D_{+}\right) /\left(D_{+}-D_{-}\right) \quad \text { and } \quad \delta=\log (s / r) /\left(D_{+}-D_{-}\right) .
$$

The result then follows on invoking Lemma 5

\section{Numerical eVAluation OF CERTAin CONSTANTS}

For our proof of Theorem 11 we need to evaluate the constants $C_{b_{1}}, C_{b_{3}}, B_{b_{1}}$ and $B_{b_{3}}$ with enough numerical precision. The purpose of this section is to achieve this. (Recall that $B_{b_{i}}=\lim _{x \rightarrow \infty}\left(\sum_{n<x} \Lambda_{b_{i}}(n) / n-(\log x) / 2\right)$.)

We first consider the evaluation of $C_{b_{3}}$ and $C_{b_{1}}$ (defined by (111)). We have, for $\operatorname{Re}(s)>1$,

$$
L_{b_{3}}(s)=\left(1-3^{-s}\right)^{-1} \prod_{p \equiv 1(\bmod 3)}\left(1-p^{-s}\right)^{-1} \prod_{p \equiv 2(\bmod 3)}\left(1-p^{-2 s}\right)^{-1}
$$

and

$$
L_{b_{3}}(s)^{2}=\zeta(s) L\left(s, \chi_{-3}\right)\left(1-3^{-s}\right)^{-1} \prod_{p \equiv 2(\bmod 3)}\left(1-p^{-2 s}\right)^{-1} .
$$

From this, (11), $\lim _{s \rightarrow 1+0}(s-1) \zeta(s)=1$ and the fact that $\Gamma\left(\frac{1}{2}\right)=\sqrt{\pi}$, we obtain

$$
C_{b_{3}}^{2}=\frac{3 L\left(1, \chi_{-3}\right)}{2 \pi} \prod_{p \equiv 2(\bmod 3)}\left(1-p^{-2}\right)^{-1},
$$

where for any fundamental discriminant $D, \chi_{D}$ denotes Kronecker's extension $(D / n)$ of the Legendre symbol [6, Chapter 5]. If $\chi$ is a real primitive character modulo $k$ and $\chi(-1)=-1$, then

$$
L(1, \chi)=-\frac{\pi}{k^{3 / 2}} \sum_{n=1}^{k} n \chi(n)
$$

by Dirichlet's celebrated class number formula (cf. equation (17) of [6, Chapter 6]). We infer that $L\left(1, \chi_{-3}\right)=\pi / \sqrt{27}$. Using that $C_{b_{3}}$ must be positive and $\zeta(2)=\pi^{2} / 6$, we then infer that

$$
C_{b_{3}}=\frac{1}{\sqrt{2}} \frac{1}{3^{1 / 4}} \prod_{p \equiv 2(\bmod 3)}\left(1-p^{-2}\right)^{-1 / 2}=\frac{\pi 3^{1 / 4} \sqrt{2}}{9} \prod_{p \equiv 1(\bmod 3)}\left(1-p^{-2}\right)^{1 / 2} .
$$

Likewise, using that $L\left(1, \chi_{-4}\right)=\pi / 4$, we find formula (2) for $C_{b_{1}}$.

Note that, for $\Re(s)>1 / 2$,

$$
\prod_{p \equiv 3(\bmod 4)}\left(1-p^{-2 s}\right)^{-2}=\frac{\zeta(2 s)\left(1-2^{-2 s}\right)}{L\left(2 s, \chi_{-4}\right)} \prod_{p \equiv 3(\bmod 4)}\left(1-p^{-4 s}\right)^{-1} .
$$

By recursion we then find from (2) and (16) the following formula:

$$
C_{b_{1}}=\frac{1}{\sqrt{2}} \prod_{n=1}^{\infty}\left(\left(1-2^{-2^{n}}\right) \frac{\zeta\left(2^{n}\right)}{L\left(2^{n}, \chi-4\right)}\right)^{1 / 2^{n+1}},
$$


which was already known to Ramanujan [1, pp. 60-66] and Shanks [21, p. 78]. Using this expression, one computes that $C_{b_{1}}=0.76422365358922066299 \cdots$. Similarly one can show that

$$
C_{b_{3}}=\frac{1}{\sqrt{2}} \frac{1}{3^{1 / 4}} \prod_{n=1}^{\infty}\left(\left(1-3^{-2^{n}}\right) \frac{\zeta\left(2^{n}\right)}{L\left(2^{n}, \chi_{-3}\right)}\right)^{1 / 2^{n+1}},
$$

and use it to compute $C_{b_{3}}=0.63890940544534388225 \cdots$, which is in agreement with the first seven (out of eight) decimals computed for $C_{b_{3}}$ by Shanks and Schmid 22 .

On noting that, for $\operatorname{Re}(s) \geq 1$,

$$
\sum_{n=1}^{\infty} \frac{\Lambda(n)-1}{n^{s}}=-\frac{\zeta^{\prime}(s)}{\zeta(s)}-\zeta(s)
$$

and using that $\zeta(s)=1 /(s-1)+\gamma+O(s-1)$, where $\gamma$ denotes Euler's constant, is the Taylor series for $\zeta(s)$ around $s=1$, one infers that

$$
\sum_{n \leq x} \frac{\Lambda(n)}{n}=\sum_{n \leq x} \frac{1}{n}-2 \gamma+o(1)=\log x-\gamma+o(1) .
$$

Taking the logarithmic derivative of (15), one obtains

$$
-2 \frac{L_{b_{3}}^{\prime}(s)}{L_{b_{3}}(s)}=-\frac{\zeta^{\prime}(s)}{\zeta(s)}-\frac{L^{\prime}\left(s, \chi_{-3}\right)}{L\left(s, \chi_{-3}\right)}+\frac{\log 3}{3^{s}-1}+2 \sum_{p \equiv 2(\bmod 3)} \frac{\log p}{p^{2 s}-1},
$$

from which one easily infers that

$$
2 \sum_{n \leq x} \frac{\Lambda_{b_{3}}(n)}{n}=\sum_{n \leq x} \frac{\Lambda(n)}{n}-\frac{L^{\prime}\left(1, \chi_{-3}\right)}{L\left(1, \chi_{-3}\right)}+\frac{\log 3}{2}+2 \sum_{p \equiv 2(\bmod 3)} \frac{\log p}{p^{2}-1}+o(1),
$$

which yields, on invoking (17),

$$
2 B_{b_{3}}=-\gamma-\frac{L^{\prime}\left(1, \chi_{-3}\right)}{L\left(1, \chi_{-3}\right)}+\frac{\log 3}{2}+2 \sum_{p \equiv 2(\bmod 3)} \frac{\log p}{p^{2}-1} .
$$

Similarly we deduce that

$$
2 B_{b_{1}}=-\gamma-\frac{L^{\prime}\left(1, \chi_{-4}\right)}{L\left(1, \chi_{-4}\right)}+\log 2+2 \sum_{p \equiv 3(\bmod 4)} \frac{\log p}{p^{2}-1} .
$$

Note that the argument above yielded

$$
B_{b_{i}}=-\lim _{s \rightarrow 1+0}\left(\frac{L_{b_{i}}^{\prime}(s)}{L_{b_{i}}(s)}+\frac{1}{2(s-1)}\right) .
$$

This can be alternatively deduced from Serre's [20] proof of (3), cf. [11].

As to the numerical evaluation of, for example, the latter prime sum, we note that

$$
2 \sum_{p \equiv 3(\bmod 4)} \frac{\log p}{p^{2}-1}=-\left.\frac{d}{d s} \log \prod_{p \equiv 3(\bmod 4)}\left(\frac{1}{1-p^{-2 s}}\right)\right|_{s=1} .
$$


Then, applying (16) $m$ times, we obtain

$$
\begin{aligned}
\sum_{p \equiv 3(\bmod 4)} \frac{\log p}{p^{2}-1}= & \sum_{p \equiv 3(\bmod 4)} \frac{\log p}{p^{2^{m+1}}-1} \\
& +\frac{1}{2} \sum_{n=1}^{m}\left\{\frac{L^{\prime}\left(2^{m}, \chi_{-4}\right)}{L\left(2^{m}, \chi_{-4}\right)}-\frac{\zeta^{\prime}\left(2^{m}\right)}{\zeta\left(2^{m}\right)}-\frac{\log 2}{2^{2^{m}}-1}\right\} .
\end{aligned}
$$

Similarly we have

$$
\begin{aligned}
\sum_{p \equiv 2(\bmod 3)} \frac{\log p}{p^{2}-1}= & \sum_{p \equiv 2(\bmod 3)} \frac{\log p}{p^{2^{m+1}}-1} \\
& +\frac{1}{2} \sum_{n=1}^{m}\left\{\frac{L^{\prime}\left(2^{m}, \chi_{-3}\right)}{L\left(2^{m}, \chi_{-4}\right)}-\frac{\zeta^{\prime}\left(2^{m}\right)}{\zeta\left(2^{m}\right)}-\frac{\log 3}{3^{2^{m}}-1}\right\} .
\end{aligned}
$$

Using these expressions, one computes $B_{b_{1}}=0.163897318634581595856 \cdots$, and similarly $B_{b_{3}}=0.1535522449949958272447 \cdots$.

Now we can invoke [10, Theorem 4] to compute the constants $C_{b_{1}}(2)$ and $C_{b_{3}}(2)$. They are given by $C_{f}(2)=\left(1+B_{f}\right) / 2$ for $f \in\left\{b_{1}, b_{3}\right\}$. We thus find that

$$
C_{b_{1}}(2)=0.581948659317290797928 \cdots, C_{b_{3}}(2)=0.576776122497497913622 \cdots \text {. }
$$

In [22] the authors write (in our notation) " $B_{3}(x)$ remains so closely proportional to $B_{1}(x)$ that it is not clear from this data whether $C_{b_{3}}(2)>C_{b_{1}}(2)$ or $C_{b_{1}}(2)<C_{b_{3}}(2)$. It would be unlikely that they are exactly equal." We thus have resolved this matter.

The numerical data from Table 1 in conjunction with the values of $C_{b_{1}}, C_{b_{1}}(2)$ and (3) suggest that $C_{b_{1}}(3)>0$ and $C_{b_{1}}(4)<0$. Similarly it seems plausible that $C_{b_{3}}(3)>0$ and $C_{b_{3}}(4)<0$.

\section{Intermezzo: On a Claim of Ramanujan}

In the previous section we have seen that $B_{b_{3}}<\log \sqrt{3}$. This knowledge suffices to disprove a claim that was made in a celebrated, hitherto unpublished, manuscript of Ramanujan [3] on the partition and tau-functions.

Let $\tau$ denote Ramanujan's tau-function. Put $T_{n}=0$ if $3 \mid \tau(n)$ and $T_{n}=1$ otherwise. In Ramanujan's manuscript we read 3, p. 64]: "We can show by transcendental methods that

$$
\sum_{k=1}^{n} T_{k}=\frac{C}{3} \int_{1}^{n} \frac{d x}{\sqrt{\log x}}+O\left(\frac{n}{(\log n)^{r}}\right)
$$

where $r$ is any positive number and

$$
C=\frac{2^{1 / 2}}{3^{1 / 4}} \cdot \frac{1-7^{-2}}{1-7^{-3}} \cdot \frac{1-13^{-2}}{1-13^{-3}} \cdot \frac{1-19^{-2}}{1-19^{-3}} \cdots \frac{1}{\left\{\left(1-2^{-2}\right)\left(1-5^{-2}\right)\left(1-11^{-2}\right) \cdots\right\}^{1 / 2}},
$$

$2,5,11, \ldots$ being primes of the form $3 k-1$ and $7,13,19, \ldots$ being primes of the form $3 k+1$." This implies that for almost all $n, \tau(n)$ is divisible by 3 .

Using that $\tau(n) \equiv n \sigma_{1}(n)(\bmod 3)$, where $\sigma_{1}(n)$ denotes the sum of the positive divisors of $n$, it is easy to see that $T_{n}$ is multiplicative and that

$$
\sum_{n=1}^{\infty} \frac{T_{n}}{n^{s}}=\prod_{p \equiv 2(\bmod 3)} \frac{1}{1-p^{-2 s}} \prod_{p \equiv 1(\bmod 3)} \frac{1+p^{-s}}{1-p^{-3 s}} .
$$


From (19) it is not difficult to verify Ramanujan's claim regarding the value of $C$. By logarithmic differentiation we obtain from (19) that

$$
\sum_{n=1}^{\infty} \frac{\Lambda_{T}(n)}{n^{s}}=\sum_{p \equiv 2(\bmod 3)} \frac{2 \log p}{p^{2 s}-1}+\sum_{p \equiv 1(\bmod 3)}\left[\frac{\log p}{p^{s}+1}+\frac{3 \log p}{p^{3 s}-1}\right] .
$$

On comparing this series with that for $\sum_{n=1}^{\infty} \Lambda_{b_{3}}(n) n^{-s}$, it is easily seen, on using the inequality $B_{b_{3}}<\log \sqrt{3}$, that

$$
B_{T}=B_{b_{3}}-\sum_{p \equiv 1(\bmod 3)} \frac{(2 p+1) \log p}{\left(p^{2}+p+1\right)(p+1)}-\log \sqrt{3}<B_{b_{3}}-\log \sqrt{3}<0 ;
$$

indeed, we have $B_{T}=-0.53 \cdots$. This shows that

$$
\sum_{k=1}^{n} T_{k}=\frac{C}{3} \frac{n}{\sqrt{\log n}}\left(1+\frac{0.23 \cdots}{\log n}+O\left(\frac{1}{(\log n)^{1+\epsilon}}\right)\right),
$$

where $0.23 \cdots=\left(1+B_{T}\right) / 2 \neq 0.5$ (here we invoked Theorem 4 of 10 ) and $\epsilon>0$. Thus the above claim of Ramanujan is false for every $r>3 / 2$ and true for $r \leq 3 / 2$.

Note that if it were true that $B_{T}=0$, an amazing identity for Euler's constant would result. The manuscript [3] contains several further assertions of the type (18) (with 3 replaced by various other primes), all of which are disproved for $r>3 / 2$ in [11.

\section{ON THE BEHAVIOUR OF $\sum_{n \leq x} \frac{\Lambda_{b_{i}}(n)}{n}-\frac{\log x}{2}$}

Put $H_{i}(x)=\sum_{n \leq x} \Lambda_{b_{i}}(n) / n-\log \sqrt{x}$, for $i=1$ and $i=3$. A good understanding of the behaviour of $H_{i}$ is needed in order to apply our key lemma, Lemma 2 Let us define, for $i=1$ and $i=3, C_{+}\left(b_{i}\right)=\sup _{x \geq 1} H_{i}(x)$ and $C_{-}\left(b_{i}\right)=\inf _{x \geq 1} H_{i}(x)$. As in 15] we define $V(x ; d, a)=\sum_{\substack{n \leq x \leq x \\ n \equiv a(\bmod d)}} \Lambda(n) / n$. It can be shown that as $x$
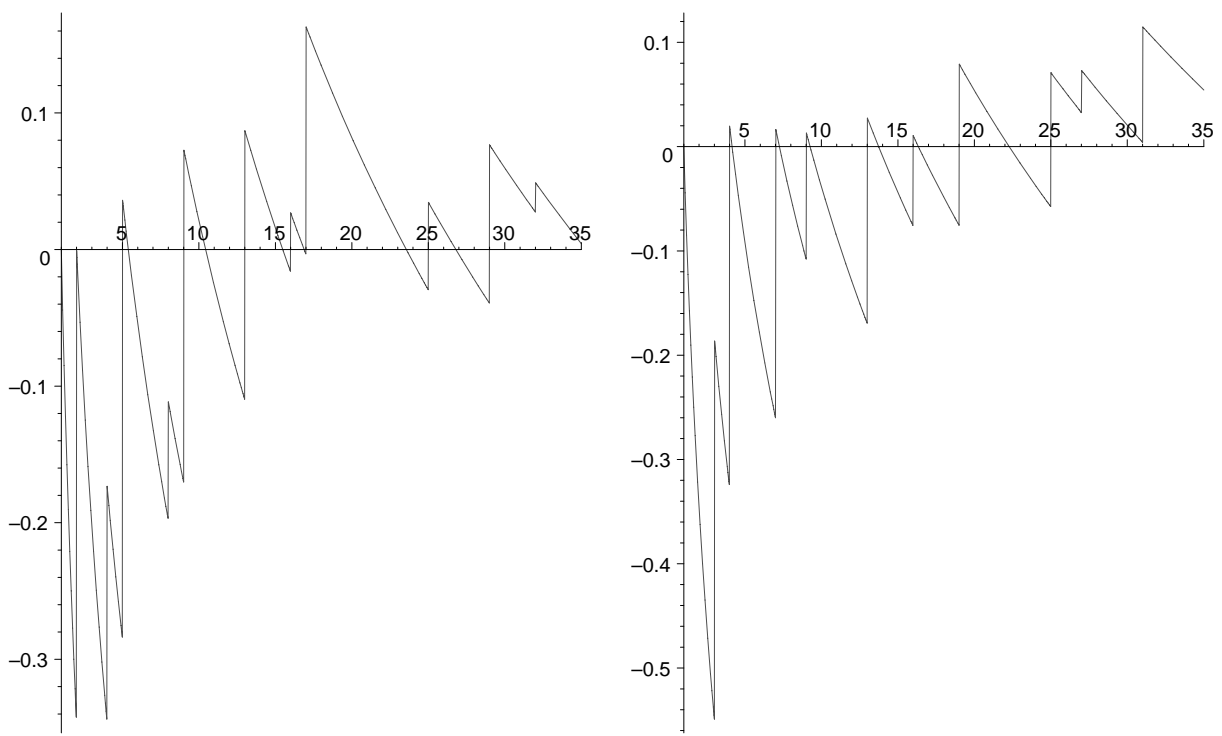

Figure 1. Plot of $H_{1}(x)$ (left) and $H_{3}(x)$ (right) for $1 \leq x \leq 35$ 
tends to infinity $V(x ; d, a)-\log x / \varphi(d)$ tends to a limit $C(d, a)$. Ramaré [15] has established the following result.

Theorem 3. [15]. For $x \geq 68$ we have $\left|V(x ; 3,1)-\frac{1}{2} \log x-C(3,1)\right| \leq 0.1205$ and $\left|V(x ; 4,1)-\frac{1}{2} \log x-C(4,1)\right| \leq 0.0961$.

We recall from 10 that

$$
\sum_{\substack{p^{r}>\sqrt{x} \\ p \equiv a(\bmod d)}} \frac{\log p}{p^{2 r}} \leq \frac{1.3}{\sqrt{x}} \text { for } x \geq 289,
$$

and that, for every fixed $v>1$ and every $x>0$,

$$
\frac{\log v}{v-1}\left(1-\frac{v}{x}\right) \leq \sum_{r=1}^{[\log x / \log v]} \frac{\log v}{v^{r}} \leq \frac{\log v}{v-1} .
$$

Theorem 4. We have:

a) $C_{-}\left(b_{1}\right)=-\log \sqrt{2}$ and $C_{+}\left(b_{1}\right)<0.2663$;

b) $C_{-}\left(b_{3}\right)=-\log \sqrt{3}$ and $C_{+}\left(b_{3}\right)<0.276$.

Proof. After some computation for the interval $[1,68]$ we infer, from Theorem 3 that $C_{+}\left(b_{1}\right) \leq B_{b_{1}}+0.0961$ and similarly $C_{+}\left(b_{3}\right) \leq B_{b_{3}}+0.1205$. For the determination of $C_{-}\left(b_{i}\right)$ we use (20) and (21) in addition to Ramaré's inequalities; this yields, for $x \geq 289$, that $H_{1}(x) \geq \frac{1}{2} \log x+B_{b_{1}}-1.3 / \sqrt{x}-(\log 4) / x$ and $H_{3}(x) \geq \frac{1}{2} \log x+B_{b_{3}}-1.3 / \sqrt{x}-(\log 27) /(2 x)$.

Let $\mathrm{RH}(d)$ be the hypothesis that for every character $\chi \bmod d$ every non-trivial zero of $L(s, \chi)$ is on the critical line.

Theorem 5. We have:

a) $C_{+}\left(b_{1}\right)=H_{1}(461)=0.1701069880305239 \cdots$, under $R H(4)$.

b) $C_{+}\left(b_{3}\right)=H_{3}(3739)=0.1554480047272349 \cdots$, under $R H(3)$.

Proof (cf. [10, Theorem 6]). We recall from [10] that for $d \leq 432$ and $(a, d)=1$, there exists a constant $c_{d, a}$ such that for $x \geq 224$ we have, under $\operatorname{RH}(d)$, that

$$
\left|\sum_{\substack{n \leq x \\ n \equiv a(\bmod d)}} \frac{\Lambda(n)}{n}-\frac{\log x}{\varphi(d)}-c_{d, a}\right| \leq \frac{11}{32 \pi \sqrt{x}}\left\{3 \log ^{2} x+8 \log x+16\right\} .
$$

Under $\mathrm{RH}(4)$ it follows from this that $C_{+}\left(b_{1}\right)=\max _{v_{i} \leq 6.15 \times 10^{8}} H_{1}\left(v_{i}\right)$, where $2=$ $v_{1}<v_{2}<\cdots$ are the consecutive prime powers that can be written as a sum of two squares. Similarly under $\mathrm{RH}(3)$ we deduce that $C_{+}\left(b_{3}\right)=\max _{w_{i} \leq 1.083 \times 10^{10}} H_{3}\left(w_{i}\right)$, where $3=w_{1}<w_{2}<\cdots$ are the consecutive prime powers that can be represented by the form $X^{2}+3 Y^{2}$. On computing these maxima (for details see Section 9), the proof is then completed.

The reason that, even under GRH, it requires a lot of computation to determine $C_{+}\left(b_{1}\right)$ and $C_{+}\left(b_{3}\right)$ is that these values are so close to $B_{b_{1}}$, respectively $B_{b_{3}}$. A similar phenomenon occurs in [10] for some of the functions considered there (cf. Theorem 6).

Using Theorem 4 and Lemma 2 together with sufficiently accurate approximations for $C_{b_{1}}$ and $C_{b_{3}}$, one infers that $\mu_{b_{1}}(x) \geq \mu_{b_{3}}(x)$ for $x \geq 27500$. After some computation we then deduce that Conjecture 3 holds true. 
Unfortunately, establishing Conjecture 2 requires quite a bit more work. In particular we need values for $D_{-}$and $D_{+}$in Lemma 2 that are closer together than those coming from Theorem 4. Without improvement of Theorem 3 the upper bounds in Theorem 4 cannot be improved. The lower bounds, however, are amenable to improvement.

Let $\Delta_{f}(x)$ denote the quantity sandwiched between $D_{-} \mu_{f}(x)$ and $D_{+} \mu_{f}(x)$ in (9). Using the lower bound for $H_{3}(x)$ appearing in the proof of Theorem 4 we deduce that $H_{3}(x) \geq 0$ for $x \geq 25$. We infer that

$$
\Delta_{b_{3}}(x) \geq-\log \sqrt{3}\left\{\mu_{b_{3}}(x)-\mu_{b_{3}}\left(\frac{x}{25}\right)\right\} .
$$

On applying Lemma 3 with $D_{-}=-\log \sqrt{3}$ and $D_{+}=0.276$, we deduce that $\Delta_{b_{3}}(x) / \mu_{b_{3}}(x) \geq-0.09586 \cdots$ for $x \geq 10^{9}$. Taking $D_{-}=-0.09586 \cdots$ as new value and repeating the procedure, we obtain $D_{-}=-0.06890 \cdots$. Iterating twice more, we see that for $x \geq 10^{9}$ we can take $D_{-}=-0.0672$ in Lemma 2 .

For any $x$ satisfying the conditions of Lemma 2, we can proceed as above. If the first iteration yields an improved value of our initial $D_{-}$(which we take to be $-\log \sqrt{3}$ ), then it is not difficult to see that every further iteration yields a value of $D_{-}$not less than the previous one (this is so since, for given $r \geq 1$, $L\left(x / r, \frac{1}{2}, D_{-}, D_{+}\right) / U\left(x, \frac{1}{2}, D_{-}, D_{+}\right)$is increasing, considered as a function in $\left.D_{-}\right)$. On the other hand, the value cannot be improved beyond zero, and hence the iteration process must converge. If the first iteration does not yield an improved value for $D_{-}$(which is initially taken as $-\log \sqrt{3}$ ), we put $\tilde{w}_{i}(x)=-\log \sqrt{3}$ for every $i \geq 0$; otherwise we put $\tilde{w}_{0}(x)=-\log \sqrt{3}$ and define

$$
\tilde{w}_{i+1}(x)=\left(\frac{L\left(\frac{x}{25} \cdot \frac{1}{2}, \tilde{w}_{i}(x), 0.276\right)}{U\left(x, \frac{1}{2}, \tilde{w}_{i}(x), 0.276\right)}-1\right) \frac{\log 3}{2} .
$$

Empirically it seems that after $n$ iterations we can expect to have approached the limit value $\lim _{i \rightarrow \infty} \tilde{w}_{i}(x)$ with $O(n)$ decimal precision.

For $b_{1}$ we proceed similarly. After some computation using the lower bound for $H_{1}(x)$ given in Theorem 4 we find that $H_{1}(x) \geq 0.065$ for $x \geq 97$. Hence

$$
H_{1}(x) \geq(-\log \sqrt{2}-0.065)\left\{\mu_{b_{1}}(x)-\mu_{b_{1}}\left(\frac{x}{97}\right)\right\}+0.065 \mu_{b_{1}}(x) .
$$

If the first iteration does not yield an improved value for $D_{-}$(which is initially taken as $-\log \sqrt{2}$ ), we put $\tilde{v}_{i}(x)=-\log \sqrt{2}$ for every $i \geq 0$; otherwise we put $\tilde{v}_{0}(x)=-\log \sqrt{2}$ and define

$$
\tilde{v}_{i+1}(x)=\left(\frac{L\left(\frac{x}{97} \cdot \frac{1}{2}, \tilde{v}_{i}(x), 0.2663\right)}{U\left(x, \frac{1}{2}, \tilde{v}_{i}(x), 0.2663\right)}-1\right)(\log \sqrt{2}+0.065)+0.065
$$

To sum up, we have established:

Lemma 6. Suppose that $x \geq x_{0} \geq 2$ and $i \geq 0$. Then 9) holds true with $f=b_{1}$, $D_{-}=\tilde{v}_{i}\left(x_{0}\right), D_{+}=0.276$. It also holds true with $f=b_{3}, D_{-}=\tilde{w}_{i}\left(x_{0}\right)$ and $D_{+}=0.2663$.

This lemma, although amenable to further improvement, is sufficiently sharp for our purposes. 


\section{The Proof of Theorem 2}

Before proving Theorem 2, we will need two more lemmas (which are illustrated in Figure 21). From prime number theory we recall that

$$
\psi(x ; d, a)=\sum_{n \leq x, n \equiv a(\bmod d)} \Lambda(n) .
$$

Lemma 7. We have:

a) $\psi_{b_{1}}(x) \geq 0.4924 x$ for $x \geq 37$.

b) $\psi_{b_{3}}(x) \leq 0.5176 x$ for $x \geq 3793$.

Proof. Let $d \leq 13$ and $(a, d)=1$. Then $|\psi(x ; d, a)-x / \varphi(d)| \leq \sqrt{x}$ for $224 \leq$ $x \leq 10^{10}$ by [16. Theorem 1] and $|\psi(x ; d, a)-x / \varphi(d)|<0.004560 x / \varphi(d)$ for $x \geq$ $10^{10}$ by [16. Theorem 5.2.1]. From these inequalities the lemma follows after some computation.

For $y \geq 3$ we define $S_{b_{3}}(y)$ by $0.5176 y$, except for the intervals $[3,49),[49,181)$, $[181,487),[487,1369),[1699,1933),[2287.2437)$, and $[3733,3793)$, where we define $S_{b_{3}}(y)$ to be (respectively) $0.653954 y, 0.605778 y, 0.557372 y, 0.534528 y, 0.526579 y$, $0.521825 y$ and $0.51996 y$.

Lemma 8. For $y \geq 2$ we have $\psi_{b_{3}}(y) \leq S_{b_{3}}(y)$.

Proof. The points where $\psi_{b_{3}}$ and $S_{b_{3}}$ change value occur only at prime powers representable by $X^{2}+3 Y^{2}$, which we denoted by $3=w_{1}<w_{2}<\cdots$. We now check that $\psi_{b_{3}}\left(w_{i}\right) \leq S_{b_{3}}\left(w_{i}\right)$ for every $w_{i} \leq 3793$. For $w_{i} \geq 3793$ the result follows by Lemma 7 .

At last we are in position to prove Theorem 2 .
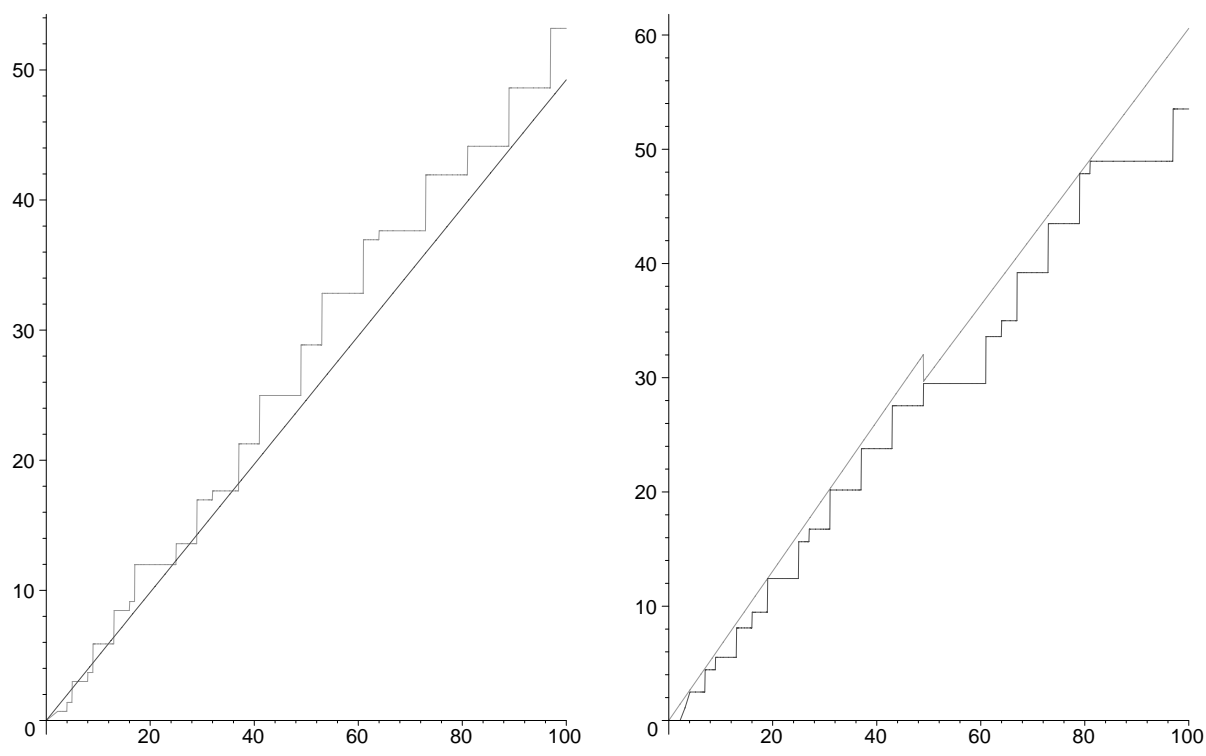

Figure 2. Plot of $\psi_{b_{1}}(x)$ versus $0.4924 x$ (left) and plot of $\psi_{b_{3}}(x)$ versus $S_{b_{3}}(x)$ (right), for $0 \leq x \leq 100$ 


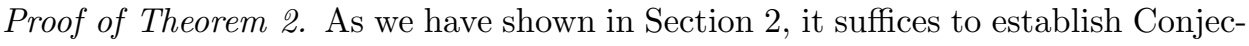
ture 2. To this end we have to prove that, for $x \geq 8$,

$$
\lambda_{b_{1}}(x)=\sum_{n \leq \frac{x}{2}} b_{1}(n) \psi_{b_{1}}\left(\frac{x}{n}\right) \geq \sum_{n \leq \frac{x}{3}} b_{3}(n) \psi_{b_{3}}\left(\frac{x}{n}\right)=\lambda_{b_{3}}(x) .
$$

Let us denote the 6 intervals in the definition of $S_{b_{3}}(y)$ by $\left[r_{i}, s_{i}\right)$ for $i=1, \ldots, 6$, and put $\alpha_{i}=S_{b_{3}}\left(r_{i}\right) / r_{i}-0.5176$ (note that $\alpha_{i}>0$ ). From Lemma 8 we infer that

$$
\lambda_{b_{3}}(x) \leq \sum_{n \leq \frac{x}{3}} b_{3}(n) S_{b_{3}}\left(\frac{x}{n}\right)=0.5176 \mu_{b_{3}}\left(\frac{x}{3}\right)+\sum_{i=1}^{6} \alpha_{i}\left\{\mu_{b_{3}}\left(\frac{x}{r_{i}}\right)-\mu_{b_{3}}\left(\frac{x}{s_{i}}\right)\right\} .
$$

Put $x_{0}=1.5 \times 10^{11}$. Using a computer (see Section 9), Conjecture 2 can be established for $x<x_{0}$. Hence, assume now that $x \geq x_{0}$. For notational convenience we shorten $U\left(x / r, \frac{1}{2}, \tilde{w}_{8}\left(x_{0} / r\right), 0.276\right)$ to $U_{3}(x / r), L\left(x / r, \frac{1}{2}, \tilde{w}_{8}\left(x_{0} / r\right), 0.276\right)$ to $L_{3}(x / r)$ and $L\left(x / r, \frac{1}{2}, \tilde{v}_{8}\left(x_{0} / r\right), 0.2663\right)$ to $L_{1}(x / r)$, where $r$ is some fixed number. On applying Lemma 6. we deduce that

$$
\frac{\lambda_{b_{3}}(x)}{2 C_{b_{3}}} \leq 0.5176 U_{3}\left(\frac{x}{3}\right)+\sum_{i=1}^{6} \alpha_{i}\left\{U_{3}\left(\frac{x}{r_{i}}\right)-L_{3}\left(\frac{x}{s_{i}}\right)\right\} .
$$

By Lemma 4 each of the six terms in the above sum is non-increasing for $x \geq x_{0}$, and thus the sum is bounded above by its value in $x_{0}$, which in its turn is less than $0.0224 U_{3}\left(x_{0} / 3\right)$. One easily checks that $U_{3}(x / 3) \geq U_{3}\left(x_{0} / 3\right)$ for $x \geq x_{0}$ (on noting that $U\left(y, \tau, D_{-}, D_{+}\right)$, considered as a function of $\tau$, is increasing for $\left.y>\exp \left(D_{+}+\left(D_{+}-D_{-}\right) / \tau\right)\right)$. We thus obtain that $\lambda_{b_{3}}(x) \leq 1.08 C_{b_{3}} U_{3}(x / 3)$. Using Lemma 6 and the lower bound for $\psi_{b_{1}}$ given in Lemma 7 we infer that $\lambda_{b_{1}}(x) \geq$ $\sum_{n \leq x / 37} b_{1}(n) \psi_{b_{1}}(x / n) \geq 0.4924 \mu_{b_{1}}(x / 37) \geq 0.9848 C_{b_{1}} L_{1}(x / 37)$. A computation shows that $0.9848 C_{b_{1}} L_{1}\left(x_{0} / 37\right)>1.08 C_{b_{3}} U_{3}\left(x_{0} / 3\right)$. By Lemma 3 we then have $0.9848 C_{b_{1}} L_{b_{1}}(x / 37)>1.08 C_{b_{3}} U_{3}(x / 3)$ for every $x \geq x_{0}$. We thus obtain that for every $x \geq x_{0}$,

$$
\lambda_{b_{1}}(x) \geq 0.9848 C_{b_{1}} L_{1}\left(\frac{x}{37}\right) \geq 1.08 C_{b_{3}} U_{3}\left(\frac{x}{3}\right) \geq \lambda_{b_{3}}(x),
$$

completing the proof.

\section{Computations of Results used in Theorems 2 and 5}

In the proof of Theorem 2 we have used the fact that Conjecture 2 is true for $x \leq x_{0}$ with $x_{0}=1.5 \times 10^{11}$. We established that result as follows.

Checking Conjecture 2 requires the computation and comparison of the sums

$$
\lambda_{b_{i}}(x)=\sum_{n \leq x} b_{i}(n) \log n, i=1,3,
$$

and, consequently, the computation of the characteristic functions $b_{1}(n)$ and $b_{3}(n)$ for all positive integers $n \leq x_{0}$. Because of the size of $x_{0}$, the range of $x$-values for which Conjecture 2 had to be checked was split up into subintervals of length $10^{6}$, large enough for efficiency, and small enough to avoid so-called cache misses during the computations. 
We first describe the case $\lambda_{b_{1}}(n)$. For a given interval, say, $[A, B]$, an integer array $b(j), j=1,2, \ldots, B-A+1$ of length $B-A+1$ is initialized to 0 . Here, $b(j)$ corresponds to $b_{1}(j+A-1)$. Next, all the possible sums of squares $x^{2}+y^{2}$ of integers $0 \leq x \leq y$, with $A \leq x^{2}+y^{2} \leq B$, hence $x \in[0, \sqrt{B / 2}], y \in[\sqrt{A / 2}, \sqrt{B}]$, are computed as follows. First, the sequence of all the squares $y^{2} \in[A / 2, B]$ is precomputed and stored. Next, for each $x=0,1, \ldots,\lfloor\sqrt{B / 2}\rfloor$, the sums $x^{2}+y^{2}$ are computed

$$
\text { for all } y^{2} \in\left[\max \left(A-x^{2}, A / 2\right), B-x^{2}\right] \subset[A / 2, B] .
$$

For all the sums $x^{2}+y^{2}=: n$ obtained in this way, $b(n-A+1)$ is set equal to 1 .

The case $\lambda_{b_{3}}(n)$ is treated similarly: the same initialization of array $b$ is carried out. Next, all the possible sums $x^{2}+3 y^{2}$ of integers $x, y$, with $A \leq x^{2}+3 y^{2} \leq B$, hence $x \in[0, \sqrt{B}], y \in[0, \sqrt{B / 3}]$, are computed as follows. First, the sequence of all the triples of squares $3 y^{2} \in[0, B]$ is precomputed and stored. Next, for each $x=0,1, \ldots,\lfloor\sqrt{B}\rfloor$, the sums $x^{2}+3 y^{2}$ are computed

$$
\text { for all } 3 y^{2} \in\left[\max \left(0, A-x^{2}\right), B-x^{2}\right] \subset[0, B]
$$

and for all the sums $x^{2}+3 y^{2}=: n$ obtained in this way, $b(n-A+1)$ is set equal to 1 . This corresponds to $b_{3}(n)$.

We have implemented these algorithms for $b_{1}(n)$ and $b_{3}(n)$ in Fortran and used them to compute $\lambda_{b_{i}}(x)$ for $i=1,3$, and to verify Conjecture 2 for $x=8,9, \ldots, 1.5 \times$ $10^{11}$ on one 250 MHZ processor of CWI's SGI Origin 2000 computing system. Computing time was 7.6 CPU hours. We also used our program to check the values of $B_{1}(x)=\sum_{n \leq x} b_{1}(n)$, given for $x=10^{i}, i=1, \ldots, 12$, by Shiu in Table 11 of [23] (where $B_{1}(x)$ is called $W(x)$ ). Computing time to extend our results from $1.5 \times 10^{11}$ to $10^{12}$ was $77 \mathrm{CPU}$ hours. We found agreement with Shiu for $i=1, \ldots, 10$, but differences for $i=11$ and $i=12: B_{1}\left(10^{11}\right)=15570512744$ and $B_{1}\left(10^{12}\right)=148736628858$, whereas Shiu gave $W\left(10^{11}\right)=15570523346$ and $W\left(10^{12}\right)=148736629005$. Shiu used a different, more efficient method than ours, but he has confirmed our value of $B_{1}\left(10^{11}\right)$ after checking and correcting his program [24].

We have spot-checked our program for computing $b_{1}(n)$ and $b_{3}(n)$ on various intervals of length $10^{6}$ with the help of Lemma 1. This requires the decomposition into primes of each $n$ for which we wish to compute $b_{1}(n)$, which is extremely expensive, compared with composing all integers in a given long interval $[A, B]$ as a sum of integer squares. However, we found agreement for all the checks we did, in particular for those in the neighbourhood of $x=10^{12}$. In Table 2, we list, for $i=1,3$, the values we found of $\lambda_{b_{i}}(x)$ and $B_{i}(x)$ for $x=j \times 10^{11}, j=1,1.5,2, \ldots, 10$.

In the proof of Theorems $5 \mathrm{a}$ and $5 \mathrm{~b}$, we have used the fact that

$$
\begin{gathered}
\max _{v_{i} \leq 6.15 \times 10^{8}} H_{1}\left(v_{i}\right)=H_{1}(461)=0.170106 \cdots, \text { respectively, } \\
\max _{w_{i} \leq 1.083 \times 10^{10}} H_{3}\left(w_{i}\right)=H_{3}(3739)=0.155448 \cdots,
\end{gathered}
$$

We established these results as follows. 
TABLE $2 . \lambda_{b_{1}}(x), B_{1}(x)$ versus $\lambda_{b_{3}}(x), B_{3}(x)$

\begin{tabular}{|c|r|r|r|r|}
\hline$x / 10^{11}$ & $\lambda_{b_{1}}(x)$ & $\lambda_{b_{3}}(x)$ & $B_{1}(x)$ & $B_{3}(x)$ \\
\hline 1 & 378458908590.818 & 316358774044.179 & 15570512744 & 13015595425 \\
1.5 & 572353849423.260 & 478438468735.511 & 23160971166 & 19360573686 \\
2 & 767521856517.400 & 641582406621.494 & 30700929088 & 25663340448 \\
3 & 1160486988190.213 & 970068358550.987 & 45678037444 & 38182949191 \\
4 & 1555965223692.576 & 1300655152892.098 & 60558145064 & 50621477125 \\
5 & 1953301629004.525 & 1632795521743.015 & 75367348255 & 63000746043 \\
6 & 2352112868630.901 & 1966168966371.294 & 90120785046 & 75333407591 \\
7 & 2752146230205.959 & 2300563843364.554 & 104828319151 & 87627692348 \\
8 & 3153223047545.408 & 2635831188875.970 & 119496904413 & 99889427349 \\
9 & 3555209733889.339 & 2971859287714.156 & 134131682979 & 112122909167 \\
10 & 3958003171956.632 & 3308561817015.470 & 148736628858 & 124331455166 \\
\hline
\end{tabular}

Let $x=6.15 \times 10^{8}$. We first generated the primes $\leq \sqrt{x}$ with the sieve of Eratosthenes, and stored the following pairs $\left(n, \Lambda_{b_{1}}(n)\right)$ :

$$
\begin{gathered}
\left(2^{k}, \log 2\right), k=1,2, \ldots,\left\lfloor\log _{2} x\right\rfloor, \\
\left(p^{2 k}, 2 \log p\right), k=1,2, \ldots,\left\lfloor\frac{1}{2} \log _{p} x\right\rfloor, \text { for the primes } p \equiv 3 \bmod 4 \leq \sqrt{x}, \\
\left(p^{k}, \log p\right), k=1,2, \ldots,\left\lfloor\log _{p} x\right\rfloor, \text { for the primes } p \equiv 1 \bmod 4 \leq \sqrt{x},
\end{gathered}
$$

into an array, sorted increasingly according to the first element of the pairs. The set of numbers $n$ in these pairs in fact contains as a subset all the prime powers $v_{1}, v_{2}, \cdots \leq \sqrt{x}$ which can be written as a sum of two squares. For these $\left(n, \Lambda_{b_{1}}(n)\right)$ pairs, we computed $H_{1}(n)$ and verified that

$$
\max _{v_{i} \leq\left\lfloor\sqrt{6.15 \times 10^{8}}\right\rfloor} H_{1}\left(v_{i}\right)=H_{1}(461)=0.170106 \cdots .
$$

The remaining interval $\left[\left\lfloor\sqrt{6.15 \times 10^{8}}\right\rfloor+1, x\right]$ was split up in pieces of length $10^{7}$, and for each of these intervals, $[A, B]$, say, the primes $p \equiv 1 \bmod 4$ were generated with the sieve of Eratosthenes, together with $\log p$. These pairs $(p, \log p)$ were mixed with the $\left(n, \Lambda_{b_{1}}(n)\right)$-pairs generated above for which $n \in[A, B]$, and then it was verified that $\max _{v_{i} \in[A, B]} H_{1}\left(v_{i}\right)<H_{1}(461)$. This proved (22). Computing time was $81 \mathrm{CPU}$ seconds. Relation (23) was proved in a similar way at the cost of 1340 CPU seconds.

\section{An ALTERNATIVE APPROACH}

In the previous sections we have made essential use of asymptotic information regarding the distribution of primes. Some of the results we used depend eventually on $\mathrm{RH}(3)$ and $\mathrm{RH}(4)$ to be true up to some finite height. It might come as a surprise then that it is possible to show that $B_{1}(x) \geq B_{3}(x)$ for $x \geq 10^{9111}$, without invoking any result from computational prime number theory (one only needs the ability to compute some successive primes...).

Our method of establishing this is inspired by Selberg's [19, pp. 183-185] method of obtaining an asymptotic evaluation for $N(x ; 4,1)$, where $N(x ; d, a)$ denotes the number of integers $n \leq x$ that have no prime factor $p$ with $p \not \equiv a(\bmod d)$. Unfortunately Selberg's method does not seem to generalise well; for example we have no idea how to generalise it so as to show that $N(x ; 4,3) \geq N(x ; 4,1)$ for $x \geq x_{0}$, with 
$x_{0}$ some effectively computable constant. See [13] for generalisations of Selberg's method.

Lemma 9. a) For $x \geq 2$ we have

$$
\left|B_{1}(x)-C_{b_{1}} \frac{x}{\sqrt{\log x}}\right| \leq 9.62 \frac{x}{\log x} .
$$

b) For $x \geq 2$ we have

$$
\left|B_{3}(x)-C_{b_{3}} \frac{x}{\sqrt{\log x}}\right| \leq 8.53 \frac{x}{\log x} .
$$

Corollary 1. For $x \geq 10^{9111}$ we have $B_{1}(x) \geq B_{3}(x)$.

In the proof of Lemma 9 we will make use of the following result. For a plot of the function $g$, see Figure 3

Lemma 10. Let $c_{2}=2 e^{\gamma}$ and $c_{3}=\sqrt{3} e^{\gamma}$. For $z \geq 1$ we put

$$
f(z)=z \sum_{n \leq z, 2 \nmid n} \frac{1}{n}-\frac{z}{2} \log \left(c_{2} z\right) \text { and } g(z)=z \sum_{n \leq z, 3 \nmid n} \frac{1}{n}-\frac{2}{3} z \log \left(c_{3} z\right) .
$$

Then

$$
\sup _{z \geq 1}|f(z)|=-f\left(3^{-}\right)=\frac{3}{2}\{\log 6+\gamma\}-3=0.55346270119438 \cdots
$$

and

$$
\sup _{z \geq 1}|g(z)|=-g\left(4^{-}\right)=\frac{8}{3} \log \left(4 \sqrt{3} e^{\gamma}\right)-6=0.70084312094794 \cdots .
$$

Proof. We only prove the statement concerning $f(z)$; the statement regarding $g(z)$ can be proved in a similar way.

Since $z \log \left(c_{2} z\right)$ is monotonically increasing, we obtain, cf. [10, Lemma 4], that $\sup _{z \geq 1}|f(z)|=\sup \left\{|f(1)|,\left|f\left(3^{-}\right)\right|,|f(3)|,\left|f\left(5^{-}\right)\right|,|f(5)|, \ldots\right\}$. Using the EulerMacLaurin summation formula, cf. [26, p. 6], one finds that for integers $n \geq 1$,

$$
\sum_{m \leq n} \frac{1}{m}=\log n+\gamma+\frac{1}{2 n}-\frac{1}{12 n^{2}}+\frac{\theta_{1}(n)}{60 n^{4}}
$$

where $\theta_{1}(n) \in[0,1]$. Clearly

$$
\sum_{m \leq n, 2 \nmid m} \frac{1}{m}=\sum_{m \leq n} \frac{1}{m}-\frac{1}{2} \sum_{m \leq n / 2} \frac{1}{m} .
$$

Let $n \geq 3$ be an odd integer. Notice that $f\left(n^{-}\right)=f(n)-1$. Using (24) and (25), it is not difficult to deduce that

$$
-\frac{1}{2} \leq f(n) \leq \frac{n}{2} \log \left(\frac{n}{n-1}\right)+\frac{n}{6(n-1)^{2}}+\frac{1}{60 n^{3}}
$$

and

$$
-\frac{1}{2} \leq-f\left(n^{-}\right) \leq \frac{1}{2}+\frac{1}{2(n-1)}+\frac{1}{12 n^{2}}+\frac{2}{15(n-1)^{4}} .
$$

Using that the latter two right-hand sides are monotonically decreasing in $n$ and noting that $\sup _{z \geq 1}|f(z)| \geq\left|f\left(3^{-}\right)\right|$, we see that $\sup _{z \geq 1}|f(z)|=\sup _{1 \leq z \leq 11}|f(z)|=$ $\left|f\left(3^{-}\right)\right|$. 


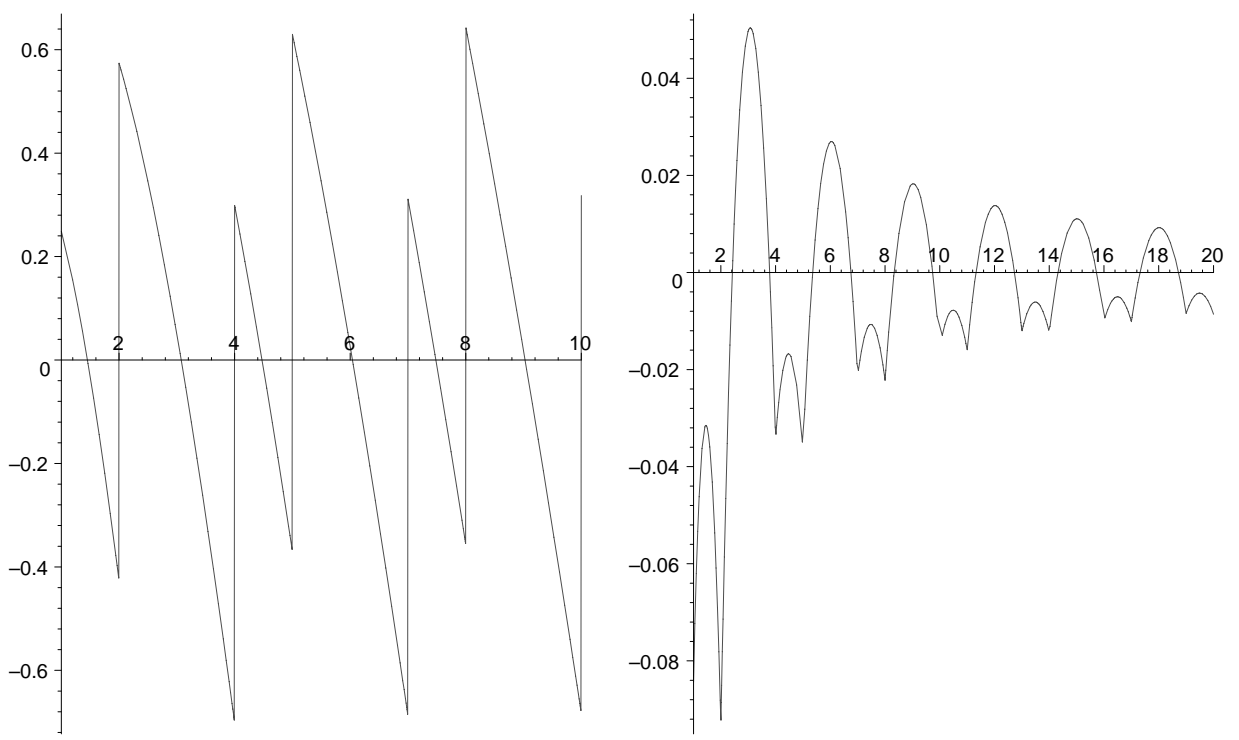

Figure 3. Plot of $g(x)$ for $1 \leq x \leq 10$ (left) and of $g(x)$ minus its limit function for $1 \leq x \leq 20$ (right)

Let $\{z\}=z-[z]$ denote the fractional part of $z$. Using (24) it can be shown that the functions $f$ and $g$ are almost periodic in the sense that they converge uniformly to the periodic functions $\frac{1}{2}-\left\{\frac{z-1}{2}\right\}$, respectively $1-\left\{\frac{z-1}{3}\right\}-\left\{\frac{z+1}{3}\right\}$ (cf. Figure 3).

\section{Proof of Lemma 9}

Let $P_{2}, P_{3}$ denote the set of primes $p$ that satisfy $p \equiv 2(\bmod 3)$, respectively $p \equiv 3(\bmod 4)$. Let $\left(P_{2}\right),\left(P_{3}\right)$ denote the set of natural numbers that have no prime divisor $p$ with $p \not \equiv 2(\bmod 3)$, respectively $p \not \equiv 3(\bmod 4)$. Let $\psi_{3}(x), \psi_{4}(x)$ denote the number of integers $1 \leq n \leq x$ that have no prime divisor $p$ with $p \not \equiv 2(\bmod 3)$, respectively $p \not \equiv 3(\bmod 4)$.

Proof of part a. Put $c_{2}=2 e^{\gamma}$. We consider the expression

$$
\begin{aligned}
& \sum_{j=0}^{\infty} \sum_{m \in\left(P_{3}\right)} \sum_{\substack{\left.1 \leq n \leq x / 2^{j} m^{2}\right) \\
n \equiv 1(\bmod 4)}} \sum_{\substack{d \mid n \\
d \in\left(P_{3}\right)}} \mu(d) \log \frac{c_{2} x}{2^{j} m^{2} d} \\
& =\sum_{j=0}^{\infty} \sum_{m \in\left(P_{3}\right)} \sum_{\substack{d \in\left(P_{3}\right) \\
d \leq x /\left(2^{j} m^{2}\right)}} \mu(d) \log \frac{c_{2} x}{2^{j} m^{2} d} \sum_{\substack{d \mid n \\
d \leq n \leq x /\left(2^{j} m^{2}\right) \\
n \equiv 1(\bmod 4)}} 1 .
\end{aligned}
$$

By approximating both sides of this equation in terms of the function $B_{1}$, we will arrive at an approximate functional equation, (34), for $B_{1}$ which on solving will yield an explicit lower bound for $B_{1}$. 
On recalling that $\sum_{d \mid n} \mu(d) \log d=-\Lambda(n)$, one sees that, when $n \equiv 1(\bmod 4)$ and $d \leq x /\left(2^{j} m^{2}\right)$, we have

$$
\sum_{\substack{d \mid n \\ d \in\left(P_{3}\right)}} \mu(d) \log \frac{c_{2} x}{2^{j} m^{2} d}= \begin{cases}\log \frac{c_{2} x}{2^{j} m^{2}} & \text { if } n \text { has no divisor } p \text { from } P_{3} ; \\ \log p & \text { if } n \text { is divisible by exactly one } p \text { from } P_{3} \\ 0 & \text { if } n \text { has } \geq 2 \text { distinct prime factors from } P_{3} .\end{cases}
$$

On noting that

$$
B_{1}(x)=\sum_{j=0}^{\infty} \sum_{m \in\left(P_{3}\right)} \psi_{4}\left(\frac{x}{2^{j} m^{2}}\right)
$$

we see that the left-hand side of (26) equals

$$
B_{1}(x) \log c_{2} x+\sum_{\substack{p \in P_{3} \\ r \geq 1}} \log p B_{1}\left(\frac{x}{p^{2 r}}\right)-\sum_{j=0}^{\infty} \sum_{m \in\left(P_{3}\right)} \log \left(2^{j} m^{2}\right) \psi_{4}\left(\frac{x}{2^{j} m^{2}}\right),
$$

which we write as

$$
B_{1}(x) \log c_{2} x+I_{1}(x)-I_{2}(x) .
$$

We write $z=x / 2^{j}$ and consider the expression formed by the three inner sums in (26), that is,

$$
\sum_{m \in\left(P_{3}\right)} \sum_{\substack{d \in\left(P_{3}\right) \\ d \leq z / m^{2}}} \mu(d) \log \frac{c_{2} z}{m^{2} d} \sum_{\substack{d \mid n, 1 \leq n \leq z / m^{2} \\ n \equiv 1(\bmod 4)}} 1 .
$$

Given an integer $k$, let $\xi(k)$ denote the product of the distinct primes that occur to an odd power in the prime factorisation of $k$. We put $\xi(k)=1$ if there is no prime that occurs to an odd power in $k$. Note that

$$
\sum_{m^{2} d=k} \mu(d)=\mu(\xi(k))
$$

where the sum is over all integers $m$ and $d$ such that $m^{2} d=k$. On writing $m^{2} d=k$ in (29), and invoking (30), we deduce that the triple sum in (29) equals

$$
\sum_{\substack{k \in\left(P_{3}\right) \\ k \leq z}} \mu(\xi(k)) \log \frac{c_{2} z}{k} \sum_{\substack{k_{1} \leq z / k \\ k_{1}=k(\bmod 4)}} 1 .
$$

The right-hand side of (26) is thus seen to equal

$$
\sum_{j=0}^{\infty} \sum_{\substack{k \in\left(P_{3}\right) \\ k \leq x / 2^{j}}} \mu(\xi(k)) \log \frac{c_{2} x}{2^{j} k} \sum_{\substack{k_{1} \leq x /\left(2^{j} k\right) \\ k_{1} \equiv k(\bmod 4)}} 1
$$

which simplifies to

$$
\sum_{\substack{d \in\left(P_{3}^{\prime}\right) \\ d \leq x}} \mu\left(\xi\left(d^{\prime}\right)\right) \log \frac{c_{2} x}{d} \sum_{\substack{k_{1} \leq x / d \\ k_{1} \equiv d^{\prime}(\bmod 4)}} 1,
$$

where $d^{\prime}$ denotes the largest odd divisor of $d$ and $P_{3}^{\prime}=P_{3} \cup\{2\}$ and $\left(P_{3}^{\prime}\right)$ is defined as $\left(P_{3}\right)$, but where now no prime divisor $p$ with $p \equiv 1(\bmod 4)$ is allowed. The 
right-hand side of (26) is thus seen to equal

$$
\sum_{\substack{d \in\left(P_{3}^{\prime}\right) \\ d \leq x}}\left[\frac{x}{4 d}+\frac{2+(-1)^{\frac{d^{\prime}-1}{2}}}{4}\right] \mu\left(\xi\left(d^{\prime}\right)\right) \log \frac{c_{2} x}{d}=\frac{x}{4} \sum_{\substack{d \in\left(P_{3}^{\prime}\right) \\ d \leq x}} \frac{\mu\left(\xi\left(d^{\prime}\right)\right)}{d} \log \frac{c_{2} x}{d}+I_{3}(x),
$$

where

$$
\left|I_{3}(x)\right| \leq \frac{3}{4} \sum_{\substack{d \in\left(P_{3}^{\prime}\right) \\ d \leq x}} \log \frac{c_{2} x}{d} .
$$

For $1 \leq d \leq x$ we have, recalling the definition of $f$ (made in Lemma 10),

$$
\frac{1}{2} \log \frac{c_{2} x}{d}=\sum_{n \leq x / d, 2 \nmid n} \frac{1}{n}-f\left(\frac{x}{d}\right) \frac{d}{x} .
$$

Combining the latter equation with the sum in the right-hand side of (31) yields

$$
\frac{x}{4} \sum_{\substack{d \in\left(P_{3}^{\prime}\right) \\ d \leq x}} \frac{\mu\left(\xi\left(d^{\prime}\right)\right)}{d} \log \frac{c_{2} x}{d}=\frac{x}{2} \sum_{\substack{d n \leq x, d \in\left(P_{3}^{\prime}\right) \\ 2 \nmid n}} \frac{\mu\left(\xi\left(d^{\prime}\right)\right)}{d n}+I_{4}(x),
$$

where

$$
\left|I_{4}(x)\right| \leq \frac{1}{2} \sum_{\substack{d \in\left(P_{3}^{\prime}\right) \\ d \leq x}}\left|f\left(\frac{x}{d}\right)\right|
$$

Note that

$$
\frac{x}{2} \sum_{\substack{d n \leq x, d \in\left(P_{3}^{\prime}\right) \\ 2 \nmid n}} \frac{\mu\left(\xi\left(d^{\prime}\right)\right)}{d n}=\frac{x}{2} \sum_{k \leq x} \frac{1}{k} \sum_{\substack{d n=k, d \in\left(P_{3}^{\prime}\right) \\ 2 \nmid n}} \mu\left(\xi\left(d^{\prime}\right)\right) .
$$

Denote the latter inner sum by $h(k)$. We claim that $h(k)=b_{1}(k)$. First let us consider the case where $k$ is odd. Then

$$
h(k)=\sum_{d n=k, d \in\left(P_{3}\right)} \mu(\xi(d))=\sum_{d n=k} \mu(\xi(d)) 2^{-\omega(d)} \prod_{p \mid d}\left(1-(-1)^{\frac{p-1}{2}}\right),
$$

where $\omega(d)$ denotes the number of distinct primes dividing $d$. We see that for odd $k, h$ is the Dirichlet convolution of two multiplicative functions and is thus itself a multiplicative function. For arbitrary $k$ we note that $h(k)=h\left(k^{\prime}\right)$, where $k^{\prime}$ is the largest odd divisor of $k$. Thus $h$ is a multiplicative function. An easy computation shows that for every prime power $q$ we have $h(q)=b_{1}(q)$. Since both $h$ and $b$ are multiplicative, this completes the proof of the claim. We thus infer that

$$
\begin{aligned}
\frac{x}{2} \sum_{\substack{d n \leq x, d \in\left(P_{3}^{\prime}\right) \\
2 \nmid n}} \frac{\mu\left(\xi\left(d^{\prime}\right)\right)}{d n} & =\frac{x}{2} \sum_{m \leq x} \frac{b_{1}(m)}{m} \\
& =\frac{x}{2} \int_{1}^{x} \frac{d B_{1}(t)}{t}=\frac{B_{1}(x)}{2}+\frac{x}{2} \int_{1}^{x} \frac{B_{1}(t)}{t^{2}} d t .
\end{aligned}
$$

Thus the right-hand side of (26) equals

$$
\frac{B_{1}(x)}{2}+\frac{x}{2} \int_{1}^{x} \frac{B_{1}(t)}{t^{2}} d t+I_{3}(x)+I_{4}(x) .
$$


Equating it with the expression (28) for the left-hand side of (26), we get

$$
B_{1}(x) \log c_{2} x-\frac{x}{2} \int_{1}^{x} \frac{B_{1}(t)}{t^{2}} d t=-I_{1}(x)+I_{2}(x)+I_{3}(x)+I_{4}(x)+\frac{B_{1}(x)}{2} .
$$

Next we will consider effective estimates for $I_{j}(x)$ for $1 \leq j \leq 4$. Using the trivial estimate $B_{1}(x) \leq x$, we obtain that

$$
0 \leq I_{1}(x) \leq x \sum_{p \in P_{3}} \frac{\log p}{p^{2}-1}<.23 x .
$$

On noting that

$$
\sum_{j=0}^{\infty} \sum_{m \in\left(P_{3}\right)} \frac{\log \left(2^{j} m^{2}\right)}{2^{j} m^{2}}=2 \sum_{m \in\left(P_{3}\right)} \frac{\log \left(2 m^{2}\right)}{m^{2}}<2.7
$$

and $\psi_{4}(x) \leq x$, we deduce that $0 \leq I_{2}(x)<2.7 x$. Using (32), we deduce that

$$
\left|I_{3}(x)\right| \leq \frac{3}{4} \sum_{d \in\left(P_{3}^{\prime}\right), d \leq x} \log \frac{c x}{d} \leq \frac{3}{4} \sum_{1 \leq d \leq x} \int_{d}^{c x} \frac{d t}{t} \leq \frac{3}{4} c x<2.68 x .
$$

For $I_{4}(x)$ we have, by (33) and Lemma 10, $\left|I_{4}(x)\right| \leq 0.277 x$.

Put $A(x)=\int_{1}^{x} B_{1}(t) d t / t^{2}$. An easy calculation (divide by $x^{2} \log ^{3 / 2} x$ and integrate) now shows that if

$$
-\alpha_{-} x \leq x^{2} \log x A^{\prime}(x)-\frac{x}{2} A(x) \leq \alpha_{+} x, \text { for } x \geq x_{0},
$$

then there exists a constant $c_{0}$ such that

$$
c_{0} \sqrt{\log x}-2 \alpha_{+} \leq A(x) \leq c_{0} \sqrt{\log x}+2 \alpha_{-} \text {, for } x \geq x_{0} .
$$

On inserting the latter estimate in (35) and invoking (1), it then follows that

$$
\left|B_{1}(x)-C_{b_{1}} \frac{x}{\sqrt{\log x}}\right| \leq\left(\alpha_{-}+\alpha_{+}\right) \frac{x}{\log x}, \text { for } x \geq x_{0} .
$$

From our estimates for $I_{j}(x)$ with $j=1, \ldots, 4$, we see that we can take $\alpha_{-}=3.96$, $\alpha_{+}=5.66$ and $x_{0}=2$.

Proof of part $b$. Making the obvious modifications in the proof of part $a$, we deduce that

$$
B_{3}(x) \log c_{3} x-\frac{x}{2} \int_{1}^{x} \frac{B_{3}(t)}{t^{2}} d t=-J_{1}(x)+J_{2}(x)+J_{3}(x)+J_{4}(x)+\frac{B_{3}(x)}{2},
$$

where

$$
\begin{gathered}
J_{1}(x)=\sum_{p \in P_{2}, r \geq 1} \log p B_{3}\left(\frac{x}{p^{2 r}}\right), \\
J_{2}(x)=\sum_{j=0}^{\infty} \sum_{m \in\left(P_{2}\right)} \log \left(3^{j} m^{2}\right) \psi_{3}\left(\frac{x}{3^{j} m^{2}}\right), \\
\left|J_{3}(x)\right| \leq \frac{2}{3} \sum_{d \in\left(P_{2}^{\prime}\right), d \leq x} \log \frac{c_{3} x}{d},
\end{gathered}
$$

and

$$
\left|J_{4}(x)\right| \leq \frac{1}{2} \sum_{d \in\left(P_{2}^{\prime}\right), d \leq x}\left|g\left(\frac{x}{d}\right)\right|,
$$


with $P_{2}^{\prime}=P_{2} \cup\{3\}$ and $\left(P_{2}^{\prime}\right)$ defined as $\left(P_{2}\right)$, but where now no prime divisor $p$ with $p \equiv 1(\bmod 3)$ is allowed. Reasoning as before, we find that

$$
0 \leq J_{1}(x)<x \sum_{p \in P_{2}} \frac{\log p}{p^{2}-1}<0.36 x
$$

and

$$
0 \leq J_{2}(x)<\frac{3}{4} x \sum_{m \in\left(P_{2}\right)} \frac{\log \left(3 m^{4}\right)}{m^{2}}<2.7 x .
$$

Furthermore we find that $\left|J_{3}(x)\right| \leq 2 x c_{3} / 3<2.06 x$ and $\left|J_{4}(x)\right| \leq 0.36 x$. From these estimates and (37) we infer that we can take $\alpha_{+}=5.12, \alpha_{-}=3.41$ and $x_{0}=2$ in the analogue of (36).

Remark. In the proof of part $a$ we have used the trivial estimates $\psi_{4}(x) \leq x$ and $B_{1}(x) \leq x$. Using that the integers $n$ counted by $\psi_{4}(x)$ satisfy $n \equiv 1(\bmod 4)$ and $3 \nmid n$, we obtain the sharper estimate

$$
\psi_{4}(x) \leq\left[\frac{x+11}{12}\right]+\left[\frac{x+7}{12}\right] \leq \frac{x}{6}+\frac{7}{6} .
$$

Similarly, some computation yields that $B_{1}(x) \leq x / 2+2$. In this way the value 9.62 appearing in Lemma $9 a$ can still be further decreased, but we have not carried this out. Similarly the estimates in the proof of part $b$ can be improved.

Acknowledgments. We thank Olivier Ramaré for giving us access to his preprint [15], some results of which were crucial in proving our main theorem. Thanks are due to Peter Shiu for comments regarding his paper [23].

\section{REFERENCES}

[1] B.C. Berndt, Ramanujan's notebooks. Part IV, Springer-Verlag, New York, $1994 . \quad$ MR 95e: 11028

[2] B.C. Berndt and R.A. Rankin, Ramanujan: Letters and commentary, AMS, Rhode Island, 1995. MR 97c:01034

[3] B.C. Berndt and K. Ono, Ramanujan's unpublished manuscript on the partition and tau functions with proofs and commentary, The Andrews Festschrift (Maratea, 1998), (Eds.) D. Foata, 2001, 39-110. MR 2000i:01027.

[4] J.H. Conway and N.J.A. Sloane, Sphere packings, lattices and groups, Third edition, Grundlehren der Mathematischen Wissenschaften 290, Springer-Verlag, New York, 1999. MR 2000b: 11077

[5] D.A. Cox, Primes of the form $x^{2}+n y^{2}$. Fermat, class field theory and complex multiplication, Wiley and Sons, Inc., New York, 1989. MR 90m:11016

[6] H. Davenport, Multiplicative number theory, Third revised edition, Springer-Verlag, New York, 2000. MR 2001f:11001

[7] S. Finch, Mathematical Constants, Cambridge University Press, to appear (2003); website http://pauillac.inria.fr/algo/bsolve/constant/constant.html.

[8] S. Kühnlein, Partial solution of a conjecture of Schmutz. Arch. Math. (Basel) 67 (1996), 164-172. MR 97h:11069

[9] E. Landau, Über die Einteilung der positiven ganzen Zahlen in vier Klassen nach der Mindestzahl der zur ihrer additiven Zusammensetzung erforderlichen Quadrate, Archiv der Math. und Physik 13 (1908), 305-312.

[10] P. Moree, Chebyshev's bias for composite numbers with restricted prime divisors, arXiv:math.NT/0112100, to appear in Math. Comp.

[11] P. Moree, On some claims in Ramanujan's 'unpublished' manuscript on the partition and tau functions, to appear in The Ramanujan Journal. 
[12] P. Moree and J. Cazaran, On a claim of Ramanujan in his first letter to Hardy, Exposition. Math. 17 (1999), 289-311. MR 2001c:11103

[13] M.R. Murty and N. Saradha, An asymptotic formula by a method of Selberg, C. R. Math. Rep. Acad. Sci. Canada 15 (1993), 273-277. MR 95d:11118

[14] A.G. Postnikov, Introduction to analytic number theory, AMS translations of mathematical monographs 68, AMS, Providence, Rhode Island, 1988. MR 89a:11001]

[15] O. Ramaré, Sur un théorème de Mertens, Manuscripta Math. 108 (2002), 495-513.

[16] O. Ramaré and R. Rumely, Primes in arithmetic progressions, Math. Comp. 65 (1996), 397425. MR 97a:11144

[17] P. Schmutz Schaller, Geometry of Riemann surfaces based on closed geodesics, Bull. Amer. Math. Soc. (N.S.) 35 (1998), 193-214. MR 99b:11098

[18] P. Schmutz Schaller, Platonische Körper, Kugelpackungen und hyperbolische Geometrie, Math. Semesterber. 47 (2000), 75-87. MR 2001m:51026

[19] A. Selberg, Collected papers, Vol. II, Springer-Verlag, Berlin, 1991. MR 95g:01032

[20] J.-P. Serre, Divisibilité de certaines fonctions arithmétiques, Enseignement Math. 22 (1976), 227-260. MR 55:7958

[21] D. Shanks, The second-order term in the asymptotic expansion of $B(x)$, Math. Comp. 18 (1964), 75-86. MR 28:2391

[22] D. Shanks and L.P. Schmid, Variations on a theorem of Landau. I, Math. Comp. 20 (1966), 551-569. MR 35:1564

[23] P. Shiu, Counting sums of two squares: the Meissel-Lehmer method, Math. Comp. 47 (1986), 351-360. MR 87h:11127

[24] P. Shiu, Private communication, February 2002.

[25] J.M. Song, Sums over nonnegative multiplicative functions over integers without large prime factors. I, Acta Arith. 97 (2001), 329-351. MR 2002f:11130

[26] G. Tenenbaum, Introduction to analytic and probabilistic number theory, Cambridge University Press, Cambridge, 1995. MR 97e:11005b

Korteweg-De Vries Institute, University of Amsterdam, Plantage Muidergracht 24, 1018 TV Amsterdam, The Netherlands

E-mail address: moree@science.uva.nl

CWI, P.O. Box 94079, 1090 GB Amsterdam, The Netherlands

E-mail address: herman@cwi.nl 